\title{
Fast Channel Allocation for Ultra-dense D2D-enabled Cellular Network with Interference Constraint in Underlaying Mode
}

\author{
Hui Dun, Fang Ye* and Shuhong Jiao \\ College of Information and Communication Engineering, Harbin Engineering University, Harbin \\ Heilongjiang Province, 150001, China \\ [e-mail: m18846198960@163.com, yefang0923@126.com, jiaoshuhong@hrbeu.edu.cn] \\ *Corresponding author: Fang Ye
}

Received September 5, 2020; revised November 8, 2020; revised December 9, 2020; accepted January 28, 2021; published June 30, 2021

\begin{abstract}
We investigate the channel allocation problem in an ultra-dense device-to-device (D2D) enabled cellular network in underlaying mode where multiple D2D users are forced to share the same channel. Two kinds of low complexity solutions, which just require partial channel state information (CSI) exchange, are devised to resolve the combinatorial optimization problem with the quality of service (QoS) guaranteeing. We begin by sorting the cellular users equipment (CUEs) links in sequence in a matric of interference tolerance for ensuring the SINR requirement. Moreover, the interference quota of CUEs is regarded as one kind of communication resource. Multiple D2D candidates compete for the interference quota to establish spectrum sharing links. Then base station calculates the occupation of interference quota by D2D users with partial CSI such as the interference channel gain of D2D users and the channel gain of D2D themselves, and carries out the channel allocation by setting different access priorities distribution. In this paper, we proposed two novel fast matching algorithms utilize partial information rather than global CSI exchanging, which reduce the computation complexity. Numerical results reveal that, our proposed algorithms achieve outstanding performance than the contrast algorithms including Hungarian algorithm in terms of throughput, fairness and access rate. Specifically, the performance of our proposed channel allocation algorithm is more superior in ultra-dense D2D scenarios.
\end{abstract}

Keywords: Ultra-dense D2D, Frequency Selective Channel, Channel Allocation, Interference Constraint, Partial CSI Exchange

This paper is funded by the International Exchange Program of Harbin Engineering University for Innovation-oriented Talents Cultivation. This paper is supported by the Fundamental Research Funds for the Central Universities under Grant 3072020CF0811 and Key Laboratory of Advanced Marine Communication and Information Technology, Ministry of Industry and Information Technology, Harbin Engineering University, Harbin, China. This work is partially supported by the Natural Science Foundation of Heilongjiang Province, China under Grant F2017004, the National Natural Science Foundation of China under Grant 61701134. 


\section{Introduction}

$\mathbf{D}_{\text {evice-to-device (D2D) communications is regarded as the key technology of the next }}$ generation mobile communication system because of its significant improvement in the energy efficiency and spectrum efficiency [1-3]. Specifically, D2D communications allow devices close to each other exchange information directly without the base station (BS) which make a shortcut in term of transmission distance and take advantage of the so-called proximity gains. Whereas, D2D communication users in underlaying mode will cause inter-tier interference to cellular users equipment (CUE) using the same channel. The situation gets worse in an ultra-dense D2D communications network where multiple D2D pairs are forced to share the same spectrum with CUE and leads to inter-tier and intra-tier interference coexisted [4-6]. Thus, the issue of channel allocation for D2D pairs with interference suppression becomes critical.

Extensive works have been studied on channel allocation for D2D enabled network. In sparse D2D communication networks, which means the quantity of D2D users are no more than quantity of CUEs, resource allocation problem of D2D users is usually modeled as a 0-1 integer combinational optimization problem [7-13]. One kind of them concentrates on the optimal allocation with an attempt to exhaustive searches, for example, Hungarian or graph theory-based algorithm [7-10]. Specifically, in such methods the BS is required to handle the global channel state information (CSI), though optimum, are computationally costly. Unlike exhaustive search, Wang et al. [11] propose a fast bi-partite graph-based matching method with two stages and the computational cost is reduced by narrowing the candidate D2D pairs set. Whereas, the above-mentioned algorithm requires the BS to have knowledge of the instantaneous CSI of all links, and the algorithm is only suitable for one-to-one matching mode. Lu et al. [13] investigated the D2D communication channel allocation problem under the Nakagami-m fading channel environment, and proposed an allocation algorithm based on weighted bipartite graph matching. When the number of D2D users and the number of cellular users do not match, the number of both is equalized by adding virtual nodes. Different from the aforementioned channel allocation algorithms that require global CSI, there are relatively few studies on channel allocation using partial channel state information in one-to-one reusing mode. In [14], the paper studies the resource allocation algorithm in the imperfect CSI environment, and proposes a joint channel allocation and power control algorithm based on location information to ensure the probability of interruption. Similarly, in [15], the author studied the spectrum efficiency optimization problem of the vehicle-to-vehicle (V2V) networking system based on D2D communication, and assumed that the BS could not grasp the fast fading information of the channel not directly connected to it, and proposed a delay CSI based resource allocation algorithm. Although the channel allocation algorithm that uses partial CSI has a loss in performance compared to the global CSI algorithm, it has significant advantages in reducing cellular network signaling consumption and reducing the computing power occupation of participating nodes such as BS. It should be pointed out that the above advantages are more obvious in the ultra-dense D2D network considered in this paper.

In ultra-dense D2D scenarios, D2D users have to adopts many-to-one or even many-to-many reusing mode to improve its access rate. Therefore, corresponding channel allocation problems in multi-user reusing mode need further study. Through analysis of existing literature, we found that more and more studies used game theory to model and analyze the channel allocation problems in ultra-dense scenarios. In general, in the process of 
D2D communication resource allocation, D2D users and cellular users use non-cooperative or cooperative ways to optimize their own communication performance, ignoring or consider the impact of this optimization on other users. Therefore, the resource allocation of the D2D communication system is essentially a game process, and a reasonable game relationship can be established between users to achieve the goal of improving the utilization rate of wireless resources and meeting users' various service quality requirements [16-21]. In [16], a price based Stackelberg game was proposed with interference constraint, which is proposed to resolve the discrete power control and channel allocation issue in a distributed manner. However, the algorithm will finally reach the convergence after 200 iterations. In [17], Penda et al. propose a new decentralized solution in which the potential game is adopted to reflect channel allocation question. The authors demonstrated that the Nash equilibrium solution of the proposed game is the local optimal solution of the problem. In [18], Militano et al. defined a constrained coalition formation game to model the channel allocation question, where each subchannel is assumed to be shared by multiple D2D users. Similarly, Wu et al. in [19] studied the problem of uplink resource sharing by many-to-many reused mode. Then, they formulated corresponding channel allocation question as a nontransferable coalition formation game. Different from the D2D resource allocation problem in the one-to-one reusing mode, in the many-to-one reusing mode, related research based on partial CSI is even more lacking. However, in an ultra-dense scenario, the computing power of the BS is higher, and the signaling consumption under the global CSI increases exponentially. Therefore, the research on channel allocation based on partial CSI has greater application prospects.

In this paper, we focus on the channel allocation question of many-to-one reusing mode for ultra-dense D2D communications network with assuming power adaption has accomplished rather than the coupled problem of power control and channel allocation. The main contributions of this paper are as follows:

- Different from existing channel allocation algorithms, we develop two novel fast channel allocation algorithms that require partial CSI exchanging for BS and avoid signaling overhead within an ultra-dense D2D communications network. Specifically, this paper investigate optimization problems with maximizing system throughput as the objective function with assuming multiple D2D pairs are permitted to share the same channel.

- In particular, to ensure the QoS of CUEs, the BS evaluates interference tolerance for each channel in terms of the SINR threshold and announces to all D2D pairs. Furthermore, multiple D2D pairs compete for the interference quota in each frequency-selective channel where the number of available channels is far less when compare to the quantity of D2D users. Moreover, we will investigate how to optimize channel allocation between the CUEs side and the D2D pairs side.

Our arrangement for the rest of the article is as follows. Section II presents the interference model of cellular network under ultra-dense D2D communications and describes the channel allocation problem under the model considered. Then the proposed channel allocation algorithms with partial information are discussed in In Section III. In Sections IV, we present the simulation results and followed by a conclusion given in Section V. The key notations and variables used in this paper are listed in Table 1. 
Table 1. List of key notations and variables

\begin{tabular}{cc}
\hline Symbols & Meaning \\
$M$ & Number of CUEs \\
$C$ & Number of D2D pairs \\
$D$ & Set of CUEs \\
$g_{i j}$ & Set of D2D pairs \\
$h_{j i}$ & Channel gain between transmitter $i$ to receiver $j$ \\
$\sigma_{N}^{2}$ & Interference channel gain between transmitter $j$ to receiver $i$ \\
$f(i, j)$ & Background noise power \\
$P_{i}$ & Channel allocation function \\
$P_{j}$ & Transmit power of CUE \\
$\gamma_{\text {th }}$ & Transmit power of D2D \\
$I_{i}^{\text {th }}$ & Minimum SINR requirement for CUE \\
$C_{\text {size }}$ & Interference tolerance for CUE \\
\hline
\end{tabular}

\section{Interference Model and Problem Description}

In this work, we investigate the channel allocation question of D2D enabled cellular network with underlaying mode, subject to CUEs minimal QoS guaranteeing, where ultra-dense D2D pairs with the number of $M$ coexisting with $N$ CUEs which occupy $N$ orthogonal resource blocks (RBs). Here, we limit our scope into a single cell where the channel allocation for CUEs has been finished and the D2D pairs are assumed to share uplink frequency resources. We utilize $D=\{1, \ldots, M\}$ and $C=\{1, \ldots, N\}$ to describe sets of D2D pairs and CUEs correspondingly and $M$ are larger than $N$. Suppose that the D2D pairs autonomously competing for $N$ channels and every D2D pair could choose no more than single CUE subchannel at any point of time. The considered network is shown in Fig. 1 which illustrates the intra-tier and inter-tier interference in the ultra-dense D2D scenario.

We assume that all channels suffer from large-scale path loss and small-scale Rayleigh fading. Specifically, the channel gains between CUEs and BS and D2D links are given by

$$
g_{i j}=\kappa \omega_{i j} d_{i j}^{-\alpha},
$$

where $\kappa$ and $\alpha$ denote the pathloss constant and pathloss exponent respectively. $d_{i j}$ is the distance from transmitter $i$ to receiver $j$. $\omega_{i j}$ is random gain due to Rayleigh fading. Similarly, the channel gains of interference links from D2D pairs to BS and CUEs towards to D2D receivers are denoted by $h_{i j}$. Hence, the SINR for CUE at the BS side is given by 


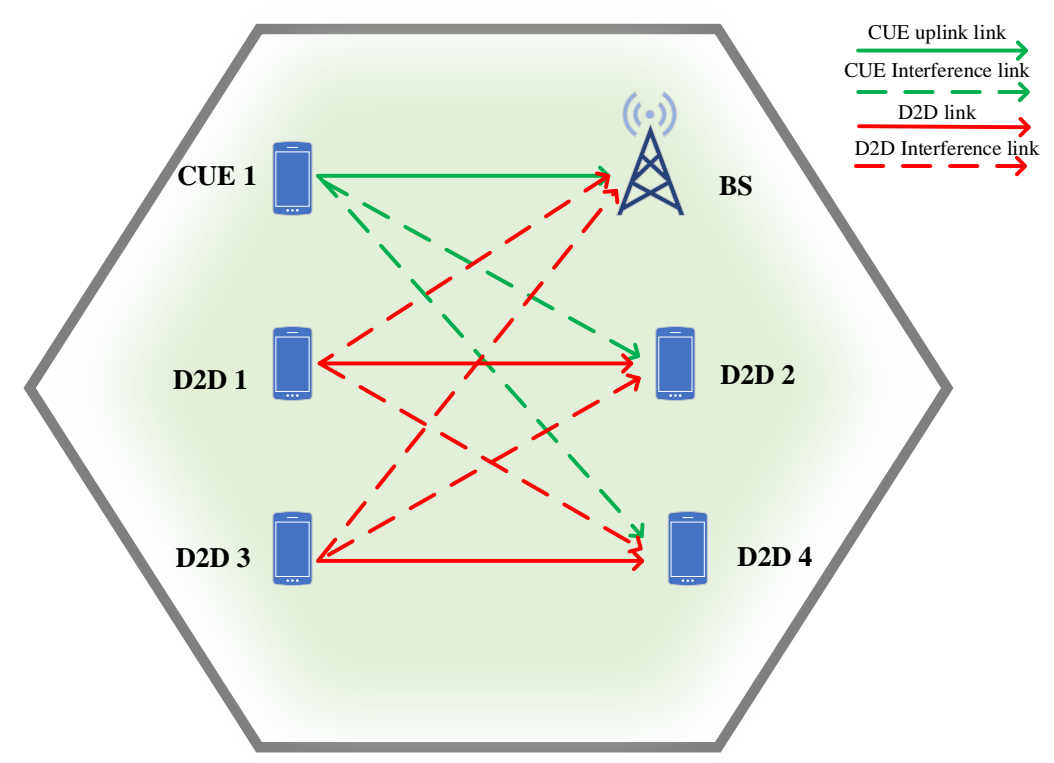

Fig. 1. Interference model of ultra-dense D2D communications with underlaying cellular network

$$
\gamma_{i}^{c}=\frac{P_{i} g_{i B}}{\sum_{j=1}^{D} P_{j} h_{j i} f(i, j)+\sigma_{N}^{2}},
$$

where $g_{i B}$ is the channel gain from CUE $i$ to BS. $P_{i}$ and $P_{j}$ are transmit power of CUE and D2D pair $j . h_{j i}$ is the interference gain between D2D pair $j$ and CUE $i . \sigma_{N}^{2}$ is the background noise. $f(i, j)$ denotes as channel allocation function which is set to be 1 when D2D link $j$ reuse CUE $i$ subchannel, otherwise 0 . We assume $f(i, j)$ satisfies

$$
\sum_{i} f(i, j) \leq 1 \forall j=1, \ldots, M,
$$

In D2D-enabled cellular network, CUEs are seen as primary users which should have the minimum QoS guaranteeing denoted by $\gamma_{i}^{c} \geq \gamma_{\text {th }}$. Unlike the sparse D2D communications network as done in [19], we set a different interference tolerance for each cellular user according to the channel gain of each user, in order to ensure the minimum QoS of each user. Under this assumption, the interference tolerance of cellular users is regarded as a virtual resource, and D2D users compete for interference quota through bidding. This assumption is based on that the CUEs will have diverse QoS requirement and the undesirable interference caused by spectrum-sharing of D2D pairs is untransferable. These ideas of diverse interference temperature constraint will balance the objective QoS of the CUEs. Herein, the above characteristics make the channel allocation problem considered in this paper different from the existing literature on the assumption of interference constraints [22].

Hence we define the concept of interference tolerance $I_{i}^{\text {th }}$ for CUE $i$ channel as

$$
I_{i}^{\text {th }}=\max \left\{0, \gamma_{t h}^{-1}\left(P_{i} g_{i, B}-\sigma_{N}^{2} \gamma_{t h}\right)\right\} .
$$


Here, we consider maximizing the system transmission rate as the optimization goal and the maximum transmission power as the constraint condition $P_{i} \leq P_{\max }^{c}$ and $P_{j} \leq P_{\max }^{d}$. Therefore, the problem to be optimized can be formulated as

$$
\begin{gathered}
\max \sum_{i}\left[\log _{2}\left(1+\gamma_{i}^{c}\right)+\sum_{j} f(i, j) \log _{2}\left(1+\gamma_{j}^{d}\right)\right] \\
\sum_{i} f(i, j) \leq 1 \forall j=1, \ldots, M \\
\sum_{j} P_{j} h_{j i} f(i, j) \leq I_{i}^{\text {th }} \forall i=1, \ldots, N
\end{gathered}
$$

where $\gamma_{j}^{d}$ denotes the SINR of D2D pair $j$. The objective for the centralized coordinating BS aims to obtain the optimal allocation function $f(i, j)$ to maximize the sum revenue. Here, the objective is defined as the sum rate of CUEs and D2D pairs which has proved to be a non-convex combinatorial problem.

\section{Channel Allocation Algorithm with Partial Information}

It should be make clear that it's difficult to resolve the resource allocation question of ultra-dense deployment interference scenarios by finding the global optimal solutions, even assuming that BS has the instantaneous CSI of all channels. Moreover, the exhaustive search method for sparse deployment interference channels over all feasible spaces became invalid.

In this paper, the transmission of each link is assumed to be based on time slots. Moreover, each time slot is composed of two phases, signaling and transmission phase. In the signaling phase, each node completes data exchange, including algorithm iteration, control information transmission and channel state information transmission. In the transmission phase, according to the transmission parameters determined in the signaling phase, including channel allocation and transmission power, complete data transmission [23]. However, due to the centralized control of channel allocation in ultra-dense scenarios, the base station as the control center will suffered signaling overhead. Therefore, this prompts us to study the channel allocation algorithm with low complexity and low signaling overhead suitable for ultra-dense scenarios.

\subsection{Admissible Candidate Set}

We decouple the power control and channel allocation problem into two independent problems with assuming that the power adaption for all entities has been finished. The rest of the letter will focus on the channel allocation problem alone.

Recall that the CUEs have an ability of interference tolerance different from each other. It means that the CUEs with larger interference tolerance ability could allow more D2D pairs to share their channels, and vice versa. Similarly, each D2D pair will generate diversity interference when access cellular network and this diversity will result in each D2D pair inherit different probabilities to share channel resources. Moreover, the frequency selectivity of the channel will exacerbate this diversity.

Different from using a combination method that has too high algorithm complexity or even unsolvable, our approaches order the CUE uplinks in sequence according to interference 
tolerance with minimum QoS guarantee. Specifically, ordering the interference tolerance only requires BS to check the channel gain of each CUE. Firstly, different from the method illustrated in [24], we narrow admissible candidate D2D pairs set size to the purpose of reducing computational complexity and balancing interference. The candidate D2D pairs set size for CUEs is given by

$$
C_{\text {size }}=\left\lfloor\frac{M}{N}\right\rfloor \text {. }
$$

$\lfloor$ • $\rfloor$ represents integer up function.

Algorithm 1 Minimum Interference Quota Request Order (MIQRO)

1: Initialization: $P_{i}$ for CUE $i$ and $P_{j}$ for D2D pair $j$ is selected. $f(i, j)=0$ for all $i \in C, j \in D$.

2: The BS calculates $I_{i}^{\text {th }}$ and orders in ascending.

3: for $i=1: N$ do

4: $\quad$ choose the CUE $i$ with minimum $I_{i}^{\text {th }}$

5: $\quad$ for $j=1: M$ do

6: $\quad$ choose the D2D pair $j$ with minimum $h_{j i}$

7: $\quad$ if $\sum_{j} P_{j} h_{j i} \leq I_{i}^{\text {th }}$ and $j \leq C_{\text {size }}$ then

8: $\quad f(i, j)=1$

9: $\quad$ remove $\mathrm{D} 2 \mathrm{D}$ pair $j$ from all CUE candidate set

10: $\quad$ else

11: break;

12: $\quad$ end if

13: end for

14: remove CUE $i$ from set and update $N, M$

15: end for

\subsection{Novel Fast Suboptimal Channel Allocation Algorithms}

For each CUE $i$, we have calculated the interference tolerance and regulated the maximum reusing D2D pairs. The D2D pairs should compete for the interference quota for itself without hurting the CUE QoS requirement. Traditionally, D2D communications will bring in more increasing in spectrum efficiency than CUEs due to the proximity gains [25]. Therefore, in order to improve the access rate of D2D users in ultra-dense scenarios and provide a foundation for subsequent research in combination with power control algorithms, channel allocation algorithms in ultra-dense scenarios should consider allocating more D2D users for each cellular user channel. By this idea, we propose a novel fast suboptimal channel allocation algorithm: Minimum Interference Quota Request Order (MIQRO) algorithm which aims to make more D2D pairs get access to the cellular network. The procedures of MIQRO are summarized in Algorithm 1. 
It should be noted that, for the purpose of getting access to more D2D pairs for each CUE, MIQRO algorithm moves on from the CUE with the lowest interference tolerance and chooses the D2D pair with lowest inter-tier interference until the interference quota runs out. We then move down to the second CUE with the lowest interference tolerance and so on. Because the BS only requires the channel gains between CUEs and BS and the interference channel gains between D2D pairs and BS, this algorithm bring in a reduction of signaling overhead and improve allocation fairness to a certain degree.

Although our proposed MIQRO algorithm provides a better opportunity for overall matching success, the D2D pairs with high channel gain but also consuming more interference quota may fail to share channel resources. To overcome this weakness and further improve overall throughput, we propose another channel allocation algorithm by modifying MIQRO as a Maximal Ratio of Channel Gain to the Interference Order (MRCGIO) algorithm. This algorithm aims to obtain more throughput revenue by setting a high priority for D2D pairs with maximum gain to interference ratio, and the procedures are summarized in Algorithm 2.

\subsection{Algorithms Complexity Analysis}

It should be noted that, the MRCGIO algorithm also doesn't need the BS requires global CSI with improving the overall throughput without substantial access performance loss. Moreover, we examine the computation complexity: our proposed MIQRO and MRCGIO algorithms have the same complexity of $O(N+M)$. On the contrast, the Hungarian algorithm is $O(N+M)^{3}$ [26].

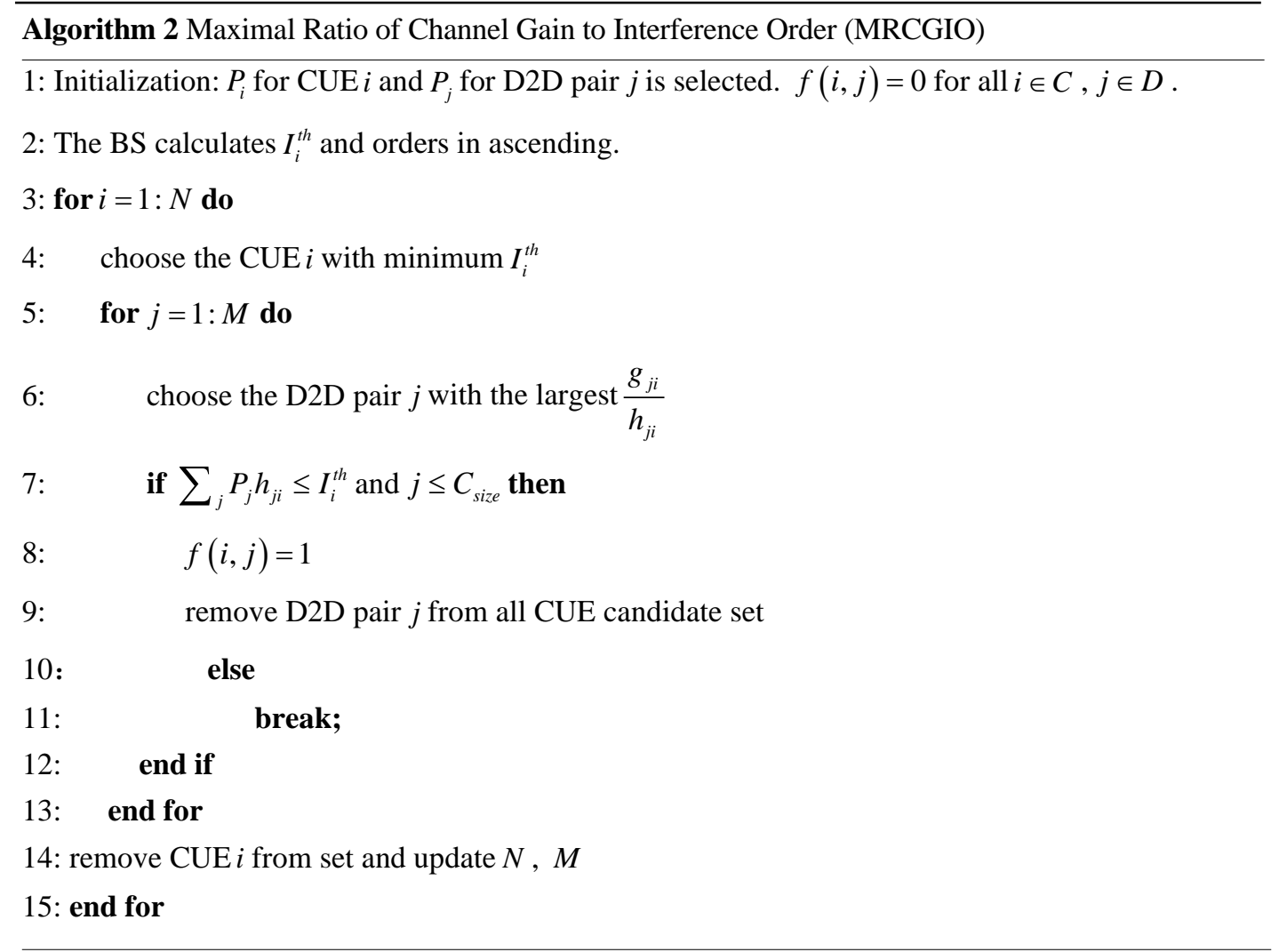




\section{Numerical Results and Analysis}

This part, we conduct experimental simulations of the proposed algorithm on the MATLAB platform, and compare the performance of the contrast algorithm to verify the advantages of the proposed algorithm in performance. Specifically, we consider the CUEs and D2D transmitters are randomly distributed within a cell. The corresponding D2D receivers are randomly distributed in a circle with the D2D transmitter as the center and the maximum transmission distance as the radius. Meanwhile, we assume the frequency selective channel environment with pathloss and fading. The specific parameters in the simulation experiment are shown in Table 2.

Table 2. Simulation parameters

\begin{tabular}{cc}
\hline Parameter & Value \\
\hline Cell radius & $500 \mathrm{~m}, 1000 \mathrm{~m}$ \\
Number of CUEs $N$ & 20 \\
Number of D2D pairs $M$ & 10 \\
D2D maximum transmission distance & $100 \mathrm{~m}$ \\
CUE maximum power $P_{\max }^{C}$ & $23 \mathrm{dBm}$ \\
D2D maximum power $P_{\max }^{D}$ & $21 \mathrm{dBm}$ \\
Noise power $\sigma_{N}^{2}$ & $-114 \mathrm{dBm}$ \\
Pathloss exponent & 3,4 \\
Shadowing & Lognormal distribution \\
Multipath fading & Exponential distribution \\
\hline
\end{tabular}

Through 1000 times Monte Carlo simulation, we demonstrate the performance superiority of our proposed two algorithms by assuming that each users' power control has finished. In this work, we focus on the channel allocation problem of the communication system and do not involve power control. Therefore, the use of fixed transmission power has no effect on the comparison of algorithm performance. Without loss of generality, the transmit power for all CUEs $i$ and D2D pairs $j$ are set to $P_{i}=P_{\max }^{C}$ and $P_{j}=0.5 P_{\max }^{D}$ respectively. We compare the proposed algorithm with random channel allocation algorithm with many-to-one reusing as well as the Hungarian allocation algorithm with one-to-one reusing which is executed along with optimal power control strategy like [9] and each D2D pair is required to reuse no more than one channel.

Fig. 2 illustrates the effects of the SINR threshold for CUEs upon overall throughput for D2D. Fig. 2 demonstrates that the overall throughput for D2D deteriorates along the upgrading of the minimum QoS requirement of CUEs in terms of SINR threshold. This because of the higher SINR threshold, the lower interference tolerance for CUEs which means less D2D pairs are eligible for sharing channel resources. Despite this, our proposed two algorithms exhibit the advantage against random algorithm and Hungarian algorithm with power control. Specifically, both of them achieve significantly sum rate improvement, and the proposed MRCGIO algorithm refine results obtained from MIQRO algorithm with allowing D2D users with higher channel gains to preferentially reuse the channel resources of CUE. At 
the same time, it can be seen from Fig. 2 that when the cell radius becomes smaller, the throughput for D2D in the many-to-one reusing mode is reduced. Moreover, the throughput performance becomes less sensitive along the increasing of the SINR threshold of CUEs. This is due to the reduction of the cell radius, which makes CUEs and D2D users in the cell more crowded, and the interference environment becomes worse. However, this also makes the transmission distance between CUEs and the BS closer, and the anti-interference ability of the CUEs are enhanced. However, the Hungarian algorithm in the one-to-one reusing mode is different from the allocation algorithm in the many-to-one reusing mode.

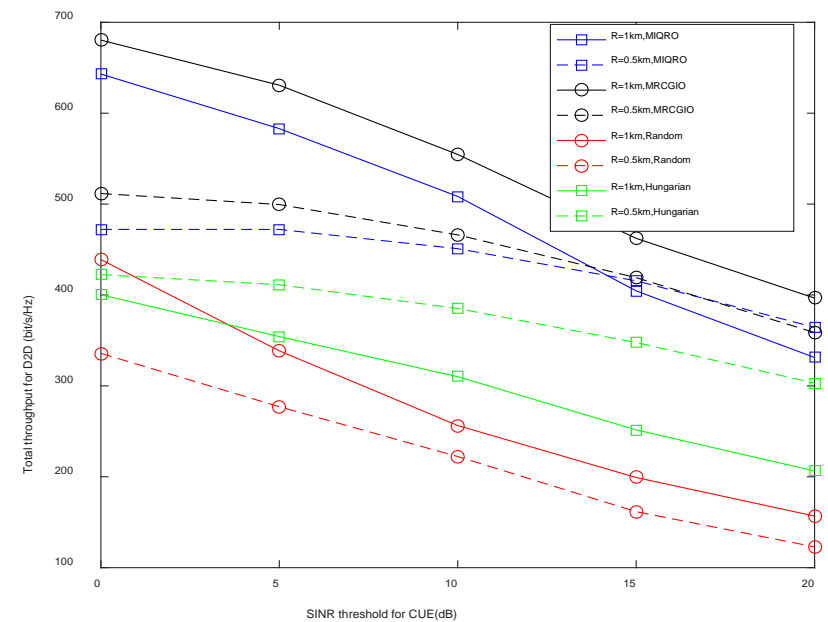

Fig. 2. Throughput for D2D with different SINR threshold of CUEs with $N=20, \quad M=100$

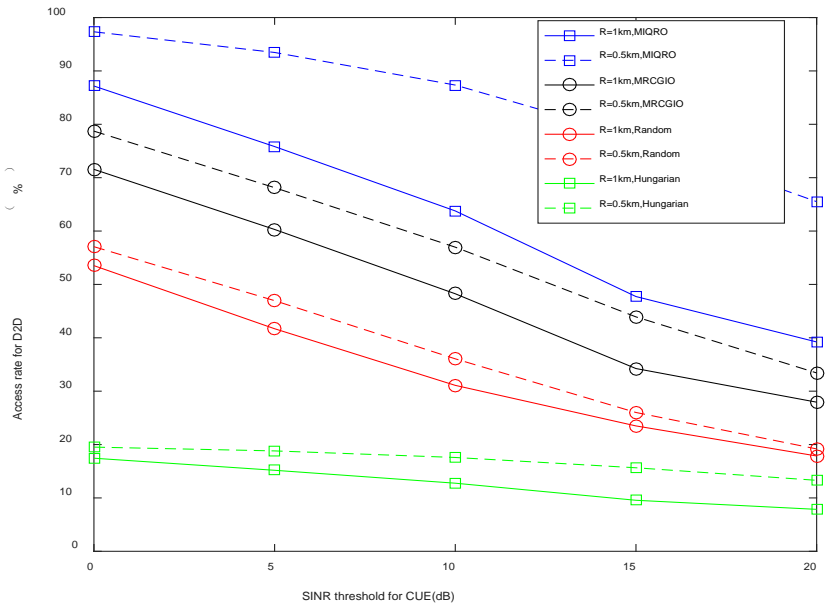

Fig. 3. Access rate for D2D with different SINR threshold of CUEs with $N=20, M=100$

Fig. 3 illustrates the effects of the SINR threshold for CUEs upon access rate for D2D. It can be seen from Fig. 3 that the two channel allocation algorithms based on partial channel state information in the many-to-one reusing mode proposed in this paper significantly improve the D2D users' access rates compared to the comparison algorithm. Moreover, Fig. 3 
verifies that the proposed channel allocation algorithms are more suitable for D2D communication networks in ultra-dense scenarios. At the same time, when the radius of the cell decreases, the access rate of D2D users is improved in different channel allocation algorithms. This is because the uplink channel gains of CUEs are improved, and the transmission rate of cellular users is well satisfied, which enhances its tolerance to D2D user interference. Different from Fig. 2, in which MRCGIO algorithm can bring more D2D user throughput than MIQRO algorithm, Fig. 3 shows that MIQRO algorithm can bring a further increase in user access rate compared to MRCGIO algorithm.

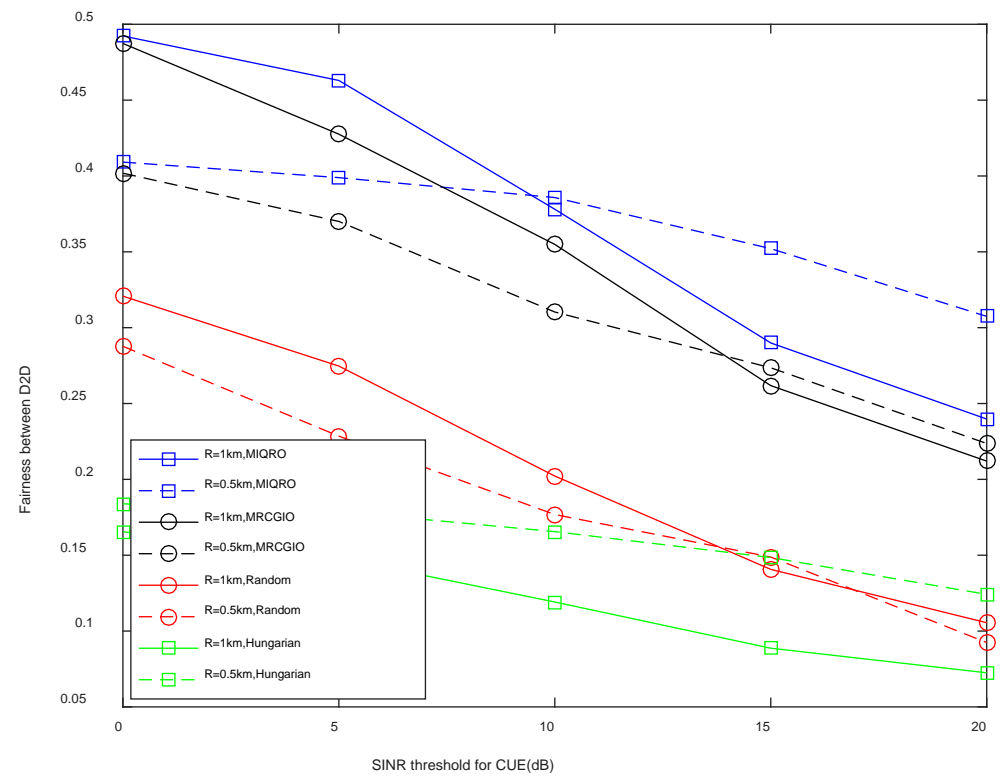

Fig. 4. Allocation fairness between D2D users with different SINR threshold for CUEs with $N=20$, $M=100$

Fig. 4 illustrates the effects of the SINR threshold for CUEs upon allocation fairness for D2D. It can be seen from Fig. 4 that the proposed MIQRO algorithm and MRCGIO algorithm have significant improvement in term of allocation fairness compared with the comparison algorithm. This is because the MIQRO algorithm and MRCGIO algorithm can enable as many users as possible to access the cellular network and reduce the difference in transmission rate between D2D users. At the same time, it can be seen from Fig. 4 that as the cell radius increases, the allocation fairness performance among D2D users becomes more sensitive with the increasing of SINR threshold for CUEs. This is due to the deterioration of the transmission performance between CUEs and BS makes CUEs only allow fewer D2D users to reuse their channel resources, thereby increasing the difference in throughput performance between D2D users.

Fig. 5 illustrates the effects of the ratio of quantity of CUEs to quantity of D2D pairs on overall throughput for D2D. Note that, both of our proposed algorithms are designed for ultra-dense D2D communications scenario. As shown in Fig. 5, by fixing $N=20$, the throughput performance for proposed MIQRO algorithm and MRCGIO algorithm bring in essential growth whereas the contrast random and Hungarian algorithm remain nearly unchanged with the increasing of D2D users density. This because CUEs channels will retain more diverse choices of potential candidate D2D pairs to reuse. Moreover, although so-called 
Hungarian algorithm can find the optimal spectrum-sharing D2D user for each channel, it is only suitable for the one-to-one reusing mode in sparse D2D communications scenarios and cannot be extended to D2D communication networks in ultra-dense scenarios.

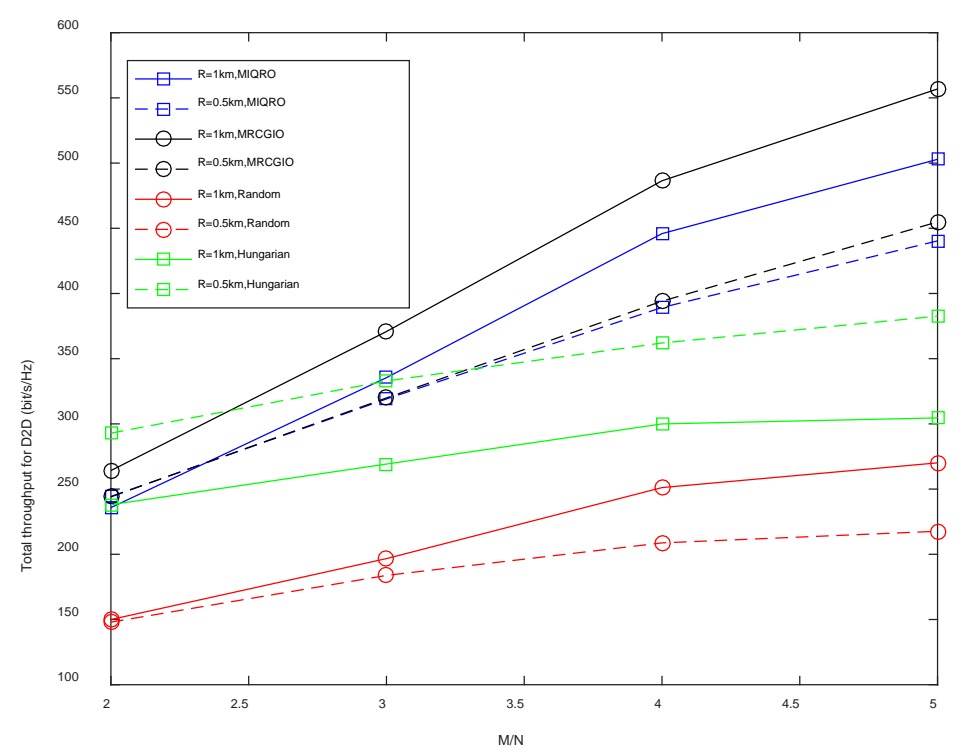

Fig. 5. Sum rate with different ratio of D2D number to CUEs with $N=20$

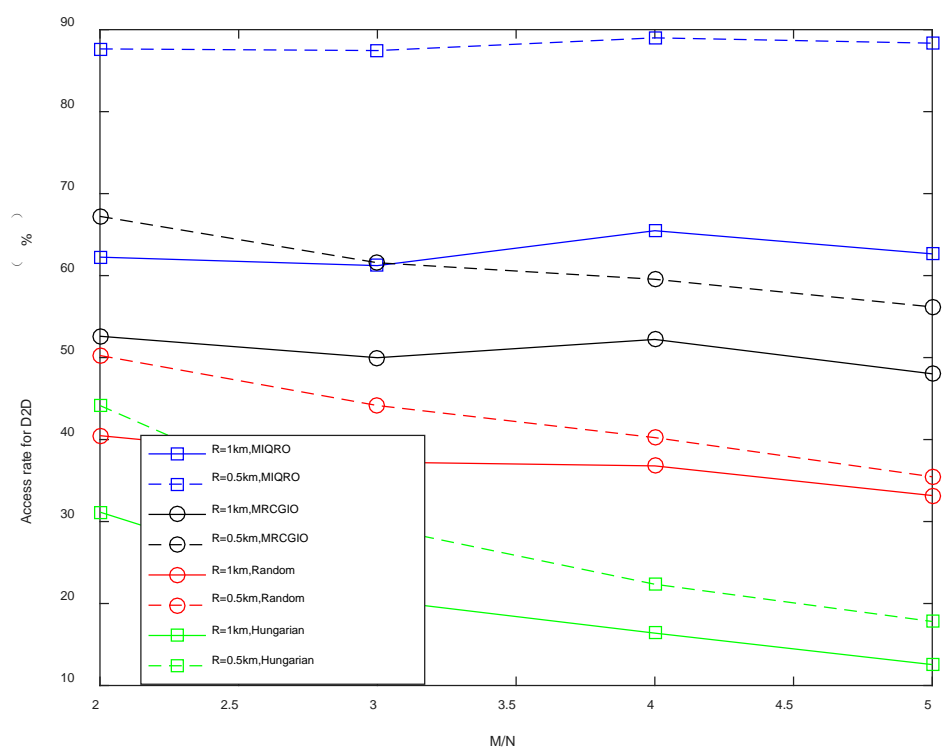

Fig. 6. Access rate for D2D users with different ratio of D2D number to CUEs with $N=20$

Fig. 6 illustrates the effects of the ratio of quantity of CUEs to quantity of D2D pairs on the access rate for D2D users. It demonstrates that our proposed MIQRO algorithm and MRCGIO algorithm improve the D2D access rate in an ultra-dense D2D communications scenario when contrast to the traditional Hungarian algorithm applied to the sparse scenario. At the same time, 
as D2D users become more and more dense, the algorithms we propose still have high user access rate when the available channel resources are consistent. This because our proposed algorithms allow D2D users with lower interference quota requirements to share channels preferentially. It also should be noted that our proposed two algorithms only require partial CSI information for BS, rather than global CSI exchange corresponding to the Hungarian algorithm. Therefore, the complexity is reduced.

\section{Conclusions}

In this work, we have conducted research on D2D communication channel allocation in ultra-dense scenarios. In order to ensure the minimum QoS requirement of cellular users, we set a maximum interference constraint for each user on the cellular user side to ensure that the total interference from D2D users to cellular users is less than the threshold. We present two novel fast channel allocation algorithms to capture the interference-constrained optimization problem with partial CSI exchange for the BS. Monte Carlo simulation shows that the two novel fast channel allocation algorithms proposed in the paper obtain significant performance improvement against contrasted Hungarian algorithm with optimal power control and the random algorithm in the metrics of throughput, allocation fairness and access rate.

\section{References}

[1] X. Kang, R. Zhang, and M. Motani, "Price-based resource allocation for spectrum-sharing femtocell networks: A Stackelberg game approach,” IEEE Journal on Selected Areas in Communications, vol. 30, no. 3, pp. 538-550, Apr. 2012. Article (CrossRef Link)

[2] R. Ansari, C. Chrysostomou, S. Hassan, M. Guizani, S. Mumtaz, J. Rodriguez, and J. Rodrigues, “5G D2D networks: techniques, challenges, and future prospects,” IEEE System Journal, vol. 12, no. 4, pp. 3970-3984, Dec. 2018. Article (CrossRef Link)

[3] N. Deng and M. Haenggi, “The benefits of hybrid caching in Gauss-Poisson D2D networks," IEEE Journal on Selected Areas in Communications, vol. 36, no. 6, pp. 1217-1230, June 2018. Article (CrossRef Link)

[4] E. Bastug, M. Bennis, and M. Debbah, "Living on the edge: The role of proactive caching in 5G wireless networks,” IEEE Communications Magazine, vol. 52, no. 8, pp. 82-89, Aug. 2014. Article (CrossRef Link)

[5] A. Antonopoulos, E. Kartsakli, and C. Verikoukis, "Game theoretic D2D content dissemination in 4G cellular networks,” IEEE Communications Magazine, vol. 52, no. 6, pp. 125-132, June 2014. Article (CrossRef Link)

[6] V. Sciancalepore, V. Mancuso, A. Banchs, S. Zaks, and A. Capone, "Enhanced content update dissemination through D2D in 5G cellular networks," IEEE Transactions on Wireless Communications, vol. 15, no. 11, pp. 7517-7530, Nov. 2016. Article (CrossRef Link)

[7] L. Namyoon, L. Xingqin, J. G. Andrews, and R. W. Heath, "Power control for D2D underlaid cellular networks: Modeling, algorithms, and analysis," IEEE Journal on Selected Areas in Communications, vol. 33, no. 1, pp. 1-13, Jan. 2015. Article (CrossRef Link)

[8] H. Nguyen, M. Hasegawa, and W. Hwang, "Distributed resource allocation for D2D communications underlay cellular networks," IEEE Communications Letters, vol. 20, no. 5, pp. 942-946, May 2016. Article (CrossRef Link)

[9] H. Kim, H. Nguyen, G. Kang, Y. Shin, and O. Shin, "Device-to-device communications underlaying an uplink SCMA system,” IEEE Access, vol. 7, no. 2, pp. 21756-21768, Feb. 2019. Article (CrossRef Link) 
[10] Z. Zhang, Y. Wu, X. Chu, and J. Zhang, "Resource allocation and power control for D2D communications to prolong the overall system survival time of mobile cells," IEEE Access, vol. 7, no. 2, pp. 17111-17124, Feb. 2019. Article (CrossRef Link)

[11] L. Wang, and H. Wu, "Fast pairing of device-to-device link underlay for spectrum sharing with cellular users,” IEEE Communications Letters, vol. 18, no. 10, pp. 1803-1807, Oct. 2014. Article (CrossRef Link)

[12] S. Liu, Y. Wu, L. Li, X. Liu, and W. Xu, “A two-stage energy-efficient approach for joint power control and channel allocation in D2D communication,” IEEE Access, vol. 7, no. 2, pp. 16940-16951, Feb. 2019. Article (CrossRef Link)

[13] B. Lu, S. Lin, J. Shi, and Y. Wang, "Resource allocation for D2D communications underlaying cellular networks over Nakagami-m fading channel,” IEEE Access, vol. 7, no. 2, pp. 21816-21825, Feb. 2019. Article (CrossRef Link)

[14] D. Feng, L. Lu, Y. Wu, G. Li, S. Li, and G. Feng, "Device-to-device communications in cellular networks,” IEEE Communications Magazine, vol. 52, no. 4, pp. 49-55, Apr. 2014. Article (CrossRef Link)

[15] L. Liang, G. Y. Li, and W. Xu, "Resource Allocation for D2D-enabled Vehicular Communications," IEEE Transactions on Communications, vol. 65, no. 7, pp. 3186-3197, July 2017. Article (CrossRef Link)

[16] S. Dominic and L. Jacob, "Distributed resource allocation for D2D communications underlaying cellular networks in time-varying environment," IEEE Communications Letters, vol. 22, no. 2, pp. 388-391, Feb. 2018. Article (CrossRef Link)

[17] D. Penda, A. Abrardo, M. Moretti, and M. Johansson, "Distributed channel allocation for D2D-enabled 5G networks using potential games,” IEEE Access, vol. 7, no. 2, pp. 11195-11208, Feb. 2019. Article (CrossRef Link)

[18] L. Militano, A. Orsino, G. Araniti, A. Molinaro, and A. Iera, “A constrained coalition formation game for multihop D2D content uploading,” IEEE Transactions on Wireless Communications, vol. 15, no. 3, pp. 2012-2024, Mar. 2016. Article (CrossRef Link)

[19] D. Wu, J. Wang, R. Hu, Y. Cai, and L. Zhou, "Energy-efficient resource sharing for mobile device-to-device multimedia communications,” IEEE Transactions on Vehicular Technology, vol. 63, no. 5, pp. 2093-2103, June 2014. Article (CrossRef Link)

[20] Y. Li, D. Jin, J. Yuan, and Z. Han, "Coalitional games for resource allocation in the device-to-device uplink underlaying cellular networks," IEEE Transactions on Wireless Communications, vol. 13, no. 7, pp. 3965-3977, July 2014. Article (CrossRef Link)

[21] C. Xu, L. Song, Z. Han, Q. Zhao, X. Wang, X. Cheng, and B. Jiao, “Efficiency resource allocation for device-to-device underlay communication systems: a reverse iterative combinatorial auction based approach,” IEEE Journal on Selected Areas in Communications, vol. 31, no. 9, pp. 348-358, Sep. 2013. Article (CrossRef Link)

[22] R. Yin, C. Zhong, G. Yu, Z. Zhang, K. Wong, and X. Chen, “Joint spectrum and power allocation for D2D communications underlaying cellular networks," IEEE Transactions on Vehicular Technology, vol. 65, no. 4, pp. 2182-2195, Apr. 2016. Article (CrossRef Link)

[23] S. Maghsudi and S. Stanczak, "Hybrid centralized-distributed resource allocation for device-to-device communication underlaying cellular networks," IEEE Transactions on Vehicular Technology, vol. 65, no. 4, pp. 2481-2495, Apr. 2016. Article (CrossRef Link)

[24] L. Liang, J. Kim, S. Jha, K. Sivanesan, and G. Y. Li, "Spectrum and power allocation for vehicular communications with delayed CSI feedback,” IEEE Wireless Communications and Letters, vol. 6, no. 4, pp. 458-461, Aug. 2017. Article (CrossRef Link)

[25] J. Yang, M. Ding, G. Mao, and Z. Lin, "Interference management in In-Band D2D underlaid cellular networks," IEEE Transactions on Cognitive Communications and Networking, vol. 5, no. 4, pp. 873-885, Dec. 2019. Article (CrossRef Link)

[26] Y. Li, Y. Liang, Q. Liu, and H. Wang, "Resources allocation in multicell D2D communications for internet of things,” IEEE Internet of Things, vol. 5, no. 5, pp. 4100-4108, Oct. 2018. Article (CrossRef Link) 
Hui Dun received his B.S. degree in Communication Engineering from Harbin Engineering University, Harbin, China in 2014. He is currently working towards the Ph.D. degree in Harbin Engineering University, Harbin, China since 2016. He has stayed the Electrical and Computer Engineering in Utah State University, Logan, USA in 2018 as a visiting scholar. His research interests include LTE, SC-FDMA technology, D2D and wireless resource management.

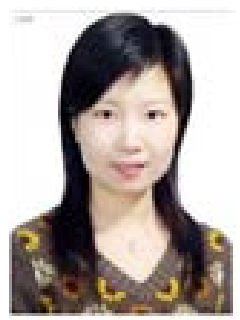

Fang Ye received the B.S. and Ph. D. degrees in Electrical Information Engineering from Harbin Engineering University(HEU) in 2002 and 2006, respectively. She has been a teacher in Harbin Engineering University of China since 2002, and became an associate professor in 2007. During 2007-2008, she stayed in School of Electronics and Computer Science from University of Southampton as a visiting scholar. Now she is an IEEE member, a member of China Institute of Communications and a member of China Computer Federation. Her research interests include LTE technology, adaptive radio resource allocation technology and Cognitive radio.

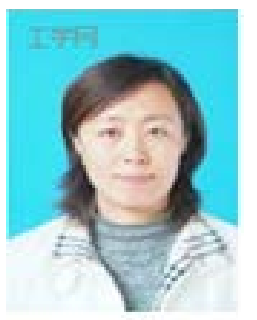

Shuhong Jiao received the B.S. and Ph. D degrees in Electrical Information Engineering from Harbin Engineering University(HEU) in 1991 and 2000, respectively. She has been a teacher in Harbin Engineering University of China since 1991, and became a professor in 2007. Now she serves as the national natural science foundation and the post-doctoral fund evaluation expert of the ministry of education. She has stayed the national institute of applied sciences in Lyon, France and Nanyang Technological University, Singapore as a visiting scholar. Her research interests include broadband signal detection, processing and identification, big data monitoring and analysis, image processing and analysis. 